\title{
Uncertainties in extracting $S_{17}$ from transfers
}

\author{
J.C. Fernandes*, R. Crespo ${ }^{\dagger}$, F.M. Nunes ${ }^{\ddagger}$ \\ Departamento de Física, Instituto Superior Técnico, \\ and Centro Multidisciplinar de Astrofísica (CENTRA) \\ Av Rovisco Pais 1096 Lisboa Codex, Portugal \\ I.J. Thompson ${ }^{\S}$ \\ Department of Physics, University of Surrey, GU2 5XH, U.K.
}

(November 21, 2018)

\begin{abstract}
The transfer reaction ${ }^{7} \mathrm{Be}(\mathrm{d}, \mathrm{n})^{8} \mathrm{~B}$ is modelled for the recent low experimental energies using the DWBA. Tests of the validity of the ANC method for the extraction of the $S_{17}$ astrophysical factor are performed. We show that a peripheral assumption is no longer accurate for center of mass energies greater than approximately $15 \mathrm{MeV}$. In all cases we found that core deformation and/or excitation effects to be small as long as the optical potential for the entrance channel is not strongly surface peaked. Multistep corrections to the DWBA were found to be small. We also emphasize that the lack of knowledge of the optical potentials for the entrance and outgoing channels induces severe uncertainties in the extracted $S_{17}$ astrophysical factor.
\end{abstract}

PACS categories: 24.10.-i, 24.10.Ht, 25.40.Cm

Typeset using REVTEX

\footnotetext{
*E-mail:jcff@wotan.ist.utl.pt

$\dagger$ E-mail:raquel@wotan.ist.utl.pt

${ }^{\ddagger}$ E-mail:filomena@wotan.ist.utl.pt

$\S$ E-mail:I.Thompson@surrey.ac.uk
} 


\section{INTRODUCTION}

The proton rich nucleus ${ }^{8} \mathrm{~B}$ has been subject to very detailed studies which are of relevance for the understanding of the so called solar neutrino problem [1]. Even if a nuclear solution cannot solve the present disagreement between the solar neutrino experiments, the $S_{17}$ astrophysical factor for the capture reaction ${ }^{7} \mathrm{Be}(\mathrm{p}, \gamma)^{8} \mathrm{~B}$ needs to be known with greater accuracy to established limits for the nonstandard theories [2].

Since the direct measurement of this reaction at the solar energies is not possible due to the hindrance by the Coulomb barrier, the measurements performed at higher energies are extrapolated (using some theoretical model) to the relevant energy regime. As published data at higher energies differ considerably from each other [3] 8 , the corresponding low energy reaction cross sections are rather ambiguous. To remove this uncertainty new measurements of the capture cross section (at the lowest possible energies) are being performed [9].

At the same time, the structure of the light nuclei involved in these capture reactions is incompletely known. The low energy behaviour of the $S_{17}$ is fairly well established now, in particular after core degrees of freedom were shown to have a negligible effect [10,11]. In contrast, the extracted normalization of the S-factor is still poorly known. It is strongly dependent on the size of the ${ }^{8} \mathrm{~B}$ nucleus and other observables [12]. If, on one hand, single particle models are suitable for predicting the low energy behaviour of the direct capture process, on the other hand they are unable to provide the normalization constant. Because of the disagreement between data sets, there is no unique determination of this normalization constant empirically from direct capture measurements.

Recently the transfer reaction has been put forward as a new tool to extract the absolute value of the $S_{17}$ factor [13] as long as the reaction is peripheral. Our aim here is to check the validity of this method.

The transfer reaction ${ }^{7} \mathrm{Be}(\mathrm{d}, \mathrm{n})^{8} \mathrm{~B}$ has been measured [14] at $\mathrm{E}_{\mathrm{cm}}=5.8 \mathrm{MeV}$ and analyzed using the DWBA zero range approximation and a simple single particle structure model. Recently new data at $\mathrm{E}_{\mathrm{cm}}=38.9 \mathrm{MeV}$ from $\mathrm{MSU}$ has become available for the same reaction [15]. The measurement of the same reaction at the centre of mass energy of $15.6 \mathrm{MeV}$ at the RIKEN Ring Cyclotron has also been performed [16].

In this work we analyze the transfer reaction ${ }^{7} \mathrm{Be}(\mathrm{d}, \mathrm{n})^{8} \mathrm{~B}$ for the relevant experimental energies using the DWBA [17]. Special attention will be paid to the peripheral assumption. The uncertainties due to the lack of knowledge of the optical potentials for the entrance and outgoing channels will be studied. The contribution of the multistep corrections to the DWBA arising from the low lying excited states in ${ }^{7} \mathrm{Be}$ will be evaluated.

\section{EXTRACTING THE $S_{17}$ FACTOR FROM TRANSFERS}

It is well established now that the direct capture S-factor $S_{17}^{\text {cap }}$ derived theoretically from nuclear structure calculations can vary considerably, and that although the energy dependence is fairly well known [11,18], the predicted astrophysical factors differ in their normalization [19]. Transfer reaction measurements, such as ${ }^{7} \mathrm{Be}(\mathrm{d}, \mathrm{n})^{8} \mathrm{~B}$ are now being used as an indirect method to extract an $S_{17}$ factor [13,14 that should not be so model dependent. 
The differential cross section for the transfer reaction has been analyzed in ref. [14] using the DWBA. In this approximation it is assumed that the ${ }^{7} \mathrm{Be}$ core remains inert in the process, and the breakup component on the deuteron wave function is only taken into account in so far that it affects the optical potential. The differential cross section is given by

$$
\frac{d \sigma}{d \Omega}=\frac{\mu_{\mathrm{i}} \mu_{\mathrm{f}}}{4 \pi^{2} \hbar^{4}} \frac{k_{n}}{k_{d}} \frac{1}{\left(2 J_{7}+1\right)\left(2 J_{d}+1\right)} \sum\left|T_{f i}\right|^{2},
$$

with $\mu_{\mathrm{i}}, \quad \mu_{\mathrm{f}}$ the reduced masses for the initial $\left({ }^{7} \mathrm{Be}-d\right)$ and final $\left({ }^{8} \mathrm{~B}-n\right)$ channels and $k_{d}, k_{n}$ the incident and outgoing momenta in the centre-of-mass frame. The transition amplitude for the transfer reaction process in the prior form is

$$
T_{f i}=\sum\left\langle\Psi_{f}^{(-)} \mathcal{I}_{{ }^{7} \mathrm{Be}^{8} \mathrm{~B}}\left|V_{n p}+V_{n^{7} \mathrm{Be}}-U_{n^{8} \mathrm{~B}}\right| \mathcal{I}_{d n} \Psi_{i}^{(+)}\right\rangle .
$$

In this equation $\Psi_{f}^{(-)}$and $\Psi_{i}^{(+)}$are the distorted waves in the final and initial channels respectively. $\mathcal{I}_{7_{\mathrm{BBe}}{ }^{8} \mathrm{~B}}$ and $\mathcal{I}_{d n}$ are the internal overlap wave functions for ${ }^{8} \mathrm{~B}\left(\mathrm{p}-{ }^{7} \mathrm{Be}\right)$ and deuteron $(\mathrm{p}-\mathrm{n})$. The remnant term, $V_{n^{7} \mathrm{Be}}-U_{n^{8} \mathrm{~B}}$, where $V_{n^{7} \mathrm{Be}}$ is the interaction between the outgoing neutron and the ${ }^{7} \mathrm{Be}$ target, is usually small and can be neglected. This term is included in our calculations but found to be small.

Within an uncorrelated two-body model for the $\mathrm{p}-{ }^{7} \mathrm{Be}$ system, the proton occupies a single particle state $n \ell j\left(\mathrm{p}_{3 / 2}\right)$ and the overlap function $\mathcal{I}_{{ }^{7} \mathrm{Be}^{8} \mathrm{~B}}$ can be defined as

$$
\mathcal{I}_{7 \mathrm{Be}^{8} \mathrm{~B}}(r)=\mathcal{S}^{1 / 2} u_{n \ell j}(r)
$$

where $\mathcal{S}$ is the spectroscopic factor that takes into account the incompleteness of the twobody model in the description of the ${ }^{8} \mathrm{~B}$ system and $u_{n \ell j}(r)$ is the normalized single particle radial function. Outside the range $R_{N}$ of the ${ }^{7} \mathrm{Be}-\mathrm{p}$ nuclear interaction, the overlap function becomes

$$
\mathcal{I}_{7_{\mathrm{Be}}{ }^{8} \mathrm{~B}} \approx \mathcal{S}^{1 / 2} b_{n \ell j} W_{\eta \ell+\frac{1}{2}}(2 \kappa r) \quad r \gg R_{N}
$$

where $W_{\eta \ell+\frac{1}{2}}(2 \kappa r)$ is the Whittaker function, $\eta=Z\left({ }^{7} \mathrm{Be}\right) e^{2} \mu / \kappa$ the Sommerfeld parameter, $\kappa=\sqrt{2 \mu \epsilon} / \hbar, \mu$ the reduced mass for the $\left(\mathrm{p}-{ }^{7} \mathrm{Be}\right)$ system, $\epsilon=0.137 \mathrm{MeV}$ the proton binding energy in ${ }^{8} \mathrm{~B}$ [20] and $b_{n l j}$ the asymptotic normalization of the single particle radial wave function $u_{n \ell j}(r)$.

The asymptotic normalization constant (ANC) for the overlap function $\mathcal{I}_{7^{\mathrm{Be}}}{ }^{8 \mathrm{~B}}$ is defined by

$$
C_{n \ell j}=\mathcal{S}^{1 / 2} b_{n \ell j}
$$

The ANC defines the probability of finding ${ }^{8} \mathrm{~B}$ in the configuration $\mathrm{p}+{ }^{7} \mathrm{Be}$ at distances outside the range of the nuclear interaction.

Following a set of calculations for different ${ }^{8} \mathrm{~B} 2$-body models, where the proton occupies a single particle state $\mathrm{p}_{3 / 2}, \mathrm{Xu}$ et al. [13] found that the ANC for the overlap function is related to the $S_{17}$ factor by

$$
S_{17}=\frac{C_{p_{3 / 2}}^{2}}{0.026}
$$


a relation that is independent of the g.s. model for ${ }^{8} \mathrm{~B}$. Using the definition of the ANC eq.(5), an astrophysical factor $S_{17}^{I}$ can therefore be determined from a spectroscopic factor and the normalization $b_{3 / 2}$ of the $\mathrm{p}^{-7} \mathrm{Be}$ wave function. The spectroscopic factor in eq.(四), is now obtained (by a $\chi^{2}$ fit) from the ratio between the data and DWBA calculations in the forward angle region, and is denoted here by $\mathcal{S}_{\exp }$. Therefore

$$
S_{17}^{I}=\frac{b_{p_{3 / 2}}^{2}}{0.026} \mathcal{S}_{\exp }
$$

This method of extracting the $S_{17}$ from transfers using the ANC will be referred as the ANC method.

We can alternatively use the direct capture calculations for extraction of the S-factor, by renormalizing the direct capture astrophysical factor $S_{17}^{\text {cap }}$ obtained from $u_{n \ell j}(r)$ by the experimental spectroscopic factor $\mathcal{S}_{\exp }$,

$$
S_{17}^{I I}=S_{17}^{\text {cap }} \mathcal{S}_{\exp }
$$

Both methods rely on at least two assumptions. They assume first that the wave function peripheral to ${ }^{8} \mathrm{~B}$ scales in proportion to the interior component, as we have written in eq.(3). Secondly, they assume that measurements of forward-angle transfer cross sections are sufficient to determine the spectroscopic factor. If DWBA analyses are used for this determination, then it is assumed further that the DWBA gives a good description for the transfer reaction, and that the distorted waves for the incoming and outgoing channels are well determined.

The ANC method of eq.(7) is only reliable when the transfer reaction is peripheral. In such cases the asymptotic part of the overlap function of eq.(因) is the dominant contributor to the transition amplitude eq.(目). For peripheral transfer reactions the results obtained through both methods are equivalent, while the method defined in eq.(8) can be used as a generalization of the ANC method when multistep effects are included in the reaction mechanism.

\section{THE ${ }^{8}$ B GROUND STATE WAVE FUNCTION}

Many two-body models have been developed for the description of ${ }^{8} \mathrm{~B}$ [21 24] that assume a spherical ${ }^{7} \mathrm{Be}$ core. The large quadrupole moment of the mirror nucleus ${ }^{7} \mathrm{Li}$ suggests however that ${ }^{7} \mathrm{Be}$ should be considerably deformed. The effects of core deformation on the capture reaction were studied in ref. [11] using a core + proton model where the core is deformed and allowed to excite. When the ${ }^{7} \mathrm{Be}$ core is assumed to be deformed then the g.s. of ${ }^{8} \mathrm{~B}\left(2^{+}\right)$will have a contribution from the $p_{1 / 2}$ proton coupled to the ${ }^{7} \mathrm{Be}\left(\frac{3}{2}^{-}\right)$channel $\left(p_{1 / 2} \otimes \frac{3}{2}^{-}\right)$, as well as $\left(p_{3 / 2} \otimes \frac{3}{2}^{-}\right)$. Within that model, if the deformation of the ${ }^{7}$ Be core is taken to be $\beta_{2}=+0.5$ the probability of finding the system in the channel $\left(p_{3 / 2} \otimes \frac{3}{2}^{-}\right)$is significantly reduced from 1.0 (no deformation) to 0.57 (reo1), while for $\beta_{2}=-0.5$ (reo2) it was found that $P\left[p_{3 / 2} \otimes \frac{3}{2}\right]=0.94$.

The ${ }^{7} \mathrm{Be}$ has a low lying excited state $\mathrm{E}\left(\frac{1}{2}^{-}\right)=0.43 \mathrm{MeV}$ and a higher spin excited state at $\mathrm{E}\left(\frac{7}{2}^{-}\right)=4.57 \mathrm{MeV}$. Due to its low excitation energy, the $\frac{1}{2}^{-}$state couples easily to the g.s. 
while the $\frac{7}{2}^{-}$state also strongly couples to the ground state. Including core excitation when calculating the g.s. of ${ }^{8} \mathrm{~B}$ enables two core excited channels that are important for the g.s.

of ${ }^{8} \mathrm{~B}$ : $\left(p_{3 / 2} \otimes \frac{1}{2}^{-}\right)$and $\left(p_{3 / 2} \otimes \frac{7}{2}^{-}\right)$. The inclusion of excitation modifies the ${ }^{8} \mathrm{~B}$ g.s., reducing the normalization of the $\left(p_{3 / 2} \otimes \frac{3}{2}^{-}\right)$channel as in the case of deformation. It was found that $\beta_{2}=+0.5$ (exc1) gives $P\left[p_{3 / 2} \otimes \frac{3}{2}\right]=0.39$, and $\beta_{2}=-0.5$ gives (exc2) $P\left[p_{3 / 2} \otimes \frac{3}{2}\right]=0.87$.

These distinct models of ${ }^{8} \mathrm{~B}$ will be used to test the validity of the ANC method, by seeing how well the extracted $S_{17}$ differs from that of the one-channel models upon the introduction of deformation/excitation and multistep core couplings.

\section{RESULTS}

\section{A. Sensitivity to the peripheral assumption}

In order to analyze the uncertainties associated with the ANC method from assuming a peripheral character for the transfer reaction, we show in fig.(1) the reaction cross section as a function of the partial wave and the corresponding differential cross section for energies $\mathrm{E}_{\mathrm{cm}}=5.8 \mathrm{MeV}, 15.6 \mathrm{MeV}$ and $38.9 \mathrm{MeV}$. The g.s. model for ${ }^{8} \mathrm{~B}$ was taken from Kim [21]. For $\mathrm{E}_{\mathrm{cm}}=5.8 \mathrm{MeV}$ we take the optical potential parameters from $\mathrm{d}-{ }^{9}$ Be elastic scattering at $\mathrm{E}_{\mathrm{cm}}=6.4 \mathrm{MeV}$ of Satchler 25] and from $\mathrm{n}{ }^{-}{ }^{9}$ Be at the correct energy of Dave and Gould [26] for the income and outgoing channel respectively. For the RIKEN experiment at $13.6 \mathrm{MeV}$ we use the potential parameters from $d-{ }^{7} \mathrm{Li}$ at $\mathrm{E}_{\mathrm{cm}}=18.7 \mathrm{MeV}$ of El-Nadi [27] and $\mathrm{n}-{ }^{9} \mathrm{Be}$ at $\mathrm{E}_{\mathrm{cm}}=19.44 \mathrm{MeV}$ of Olsson et al. [28] respectively. Since in the case of the MSU experiment at $38.9 \mathrm{MeV}$ the quasi-elastic scattering was measured for the entrance channel, we take the optical potential parameters for this channel, by extracting the elastic scattering cross section data from the quasi-elastic data [15]. For the outgoing channel we use the potential defined in ref. [28].

The transition amplitude eq.(2) involves a radial integral over the projectile-target relative distances of the incoming and outgoing distorted waves. The contribution from the outgoing channel to this integral was evaluated for radii from $\mathrm{R}_{\text {cut }}$ to infinity for several values for the cutoff radius. For $\mathrm{E}_{\mathrm{cm}}=5.8 \mathrm{MeV}$ the cross section peaks around $L=3$ corresponding to an impact parameter of $4.5 \mathrm{fm}$ which is significantly larger than the ${ }^{8} \mathrm{~B}$ interaction radius of $2.95 \mathrm{fm}$ [29] and thus, for this energy, the transfer is clearly peripheral. For the MSU energy, $\mathrm{E}_{\mathrm{cm}}=38.9 \mathrm{MeV}$, the cross section peaks for partial wave values around $L=4$ which corresponds to an impact parameter of $2.3 \mathrm{fm}$ and thus one can expect a significant contribution from the nuclear interior to the transition amplitude radial integral. This is illustrated in fig.(11) where the reaction cross section for MSU becomes significantly smaller for a cutoff radius $R_{\text {cut }}$ of the order of the nuclear radius. This contrasts with the lower energy case of $\mathrm{E}_{\mathrm{cm}}=5.8 \mathrm{MeV}$, where the contribution for the reaction cross section becomes negligible only for very large cutoff radius $R_{\text {cut }} \gg R_{N}$. It follows that, at the energies of the MSU experiment, the ANC method cannot be used by itself to extract the $S_{17}$ factor accurately. For the RIKEN experiment, the impact parameter is $3.3 \mathrm{fm}$ which is only slightly larger than the ${ }^{8} \mathrm{~B}$ interaction radius, thus caution should be taken when extracting the $S_{17}$ from the transfer reaction at this energy. 


\section{B. Sensitivity to the optical potential inputs}

The distorted waves for the incoming and outgoing channels are one of the inputs of the DWBA. The lack of measurements for the initial and final channel elastic scattering induces uncertainties in the $S_{17}$ extracted from the transfer studies, uncertainties which are accentuated when one is using elastic scattering data either at a shifted energy or on a different target (or both).

A systematic study on the optical potential uncertainties was performed recently [30,31] for the ${ }^{7} \mathrm{Be}(\mathrm{d}, \mathrm{n})^{8} \mathrm{~B}$ reaction at $\mathrm{E}_{\mathrm{cm}}=5.8 \mathrm{MeV}$. We have shown in ref. [31 that if one uses different sets of optical potential parameters for the entrance channel [14, 32, 33] that fit the elastic scattering of deuterons from ${ }^{7} \mathrm{Li}$ at higher energies $\left(\mathrm{E}_{\mathrm{cm}} \simeq 9.3 \mathrm{MeV}\right)$ the calculated transfer cross section varies considerably, producing up to $16 \%$ uncertainty in the extracted $S_{17}(0)$ (calculated relative to the mean value). This is illustrated in fig.(2) which shows that the calculated $\mathrm{d}^{-}{ }^{7} \mathrm{Be}$ elastic scattering at the correct energy $\left(\mathrm{E}_{\mathrm{cm}}=5.8 \mathrm{MeV}\right)$ using this set of potentials are different (fig.2a), even at small angles. Consequently, the calculated transfer cross section varies considerably (see fig. $2 \mathrm{~b}$ ).

Apart from the error introduced by extracting the optical potential from elastic scattering at the wrong energy, there is also an error due to the changes in the mass or charge of the target. Fig.(3) shows the calculated elastic differential cross section (fig. 3a) using deuteron optical parameters from elastic scattering on ${ }^{6} \mathrm{Li}$ and ${ }^{9} \mathrm{Be}$ at the correct energy [25, 33] and the corresponding transfer cross section (fig.3b). We find that the uncertainty on the $S_{17}(0)$ relative to its mean value is $20 \%$. For both these calculations we have used a $n-{ }^{9}$ Be potential from [26] for the exit channel and Kim's single particle potential from ${ }^{8} \mathrm{~B}$ [21].

As for the outgoing channel, the combined energy and mass/charge uncertainties in defining the optical potentials for the outgoing channel were found to be of order of $12 \%$.

If we take the whole range of optical potentials for entrance and exit channels mentioned in [31], using eq.(6), one obtains for the S-factor of $S_{17}=26.2 \pm 8.4 \mathrm{eV} \mathrm{b}$. These results show a theoretical uncertainty larger than indicated in [14] and are consistent with the recent work by the Texas A\&M group [30]. However, one can minimize this uncertainty by using only potential parameters taken from elastic scattering at the correct energy. If we reduce our set to $\mathrm{d}-{ }^{6} \mathrm{Li}$ or $\mathrm{d}-{ }^{9} \mathrm{Be}$ optical potentials from [25,33] at the correct energy for the entrance channel and n- ${ }^{9}$ Be optical potentials from [26] at the correct energy for the exit channel, we obtain $S_{17}=23.5 \pm 3.7 \mathrm{eV}$ b which indicates a smaller prediction for the S-factor than the result $S_{17}=27.4 \pm 4.4 \mathrm{eV}$ b presented in [14].

The uncertainty on the extraction of $S_{17}$ from transfer reactions induced by the lack of knowledge of the optical potentials for the incoming and outgoing channels is also evident at higher energies, such as, for example, for the RIKEN measurement. Fig. (4a) shows the calculated elastic scattering for the incoming channel using the optical potential parameters taken from $\mathrm{d}^{-7} \mathrm{Li}$ but at different center of mass energies of El-Nadi et al. at $\mathrm{E}_{\mathrm{cm}}=18.7 \mathrm{MeV}$ [27] (solid) and Matsuki et al. at $11.43 \mathrm{MeV}$ [34] (dashed). Fig.(4b) shows the corresponding transfer differential cross section. The solid and the dashed curves were evaluated using the El-Nadi and Matsuki potentials respectively for the incoming channel and the potentials of 28 for the outgoing channel. The dotted solid and dotted dashed lines were obtained using the potentials El-Nadi and Matsuki respectively for the incoming channel, but with the potentials defined in [26] for the outgoing channel. Once more, the uncertainties on the 
incoming channel have large effects on the calculated transfer reaction. The effect of the uncertainties in the outgoing channel on the transfer is comparatively less important.

\section{Sensitivity to the ${ }^{8} \mathrm{~B}$ ground state wave function}

We now study the independence of the ANC method with respect to the choice of different two-body models for the description of the ${ }^{8} \mathrm{~B}$ ground state. In particular, we study the effect of including the ${ }^{7} \mathrm{Be}$ core deformation and/or excitation. To avoid uncertainties on the calculated transfer differential cross section by modifying the incoming optical potential by distortion, we verified that elastic scattering is not significantly altered by the inclusion of deformation and/or core excitation. We show first, in fig.(5), the calculated differential cross section at $\mathrm{E}_{\mathrm{cm}}=5.8 \mathrm{MeV}$ using different single particle 2 body-models with an inert core for the description of ${ }^{8} \mathrm{~B}$. The optical potential parameters for the outgoing channel were taken to be those given in section A. The optical potential parameters for the incoming channel are taken from Bingham [33] denoted here as the Surface I potential. As follows from eqs.(5-8), the ratio of the asymptotic normalization of the single particle radial wave function to the calculated capture S-factor, $b^{2} / S_{17}^{\text {cap }}$, should be model independent and equal

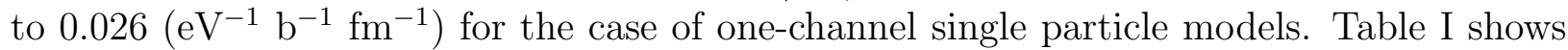
that the calculated ratio for the single particle models of Kim [21], Tombrello [22], Robertson [23], Barker [19], and Esbensen [24] are constant, in agreement with $\mathrm{Xu}$ et al. [13]. For the purpose of estimating the effect of using different ${ }^{8} \mathrm{~B}$ g.s. models, we take the astrophysical S-factor calculated for direct capture at $20 \mathrm{keV}$. In the table 【I we compare the $S_{17}^{I}$ factor evaluated for different two body models using the ANC relation eq.(17) for the data at $\mathrm{E}_{c m}=5.8 \mathrm{MeV}$, with the capture normalization constant $S_{17}^{I I}$ of eq.(8). To a good accuracy, both methods consistently show that the ANC method can deduce the $S_{17}$ from the $\mathrm{E}_{c m}=5.8$ $\mathrm{MeV}$ experiment in a manner that is essentially independent of the description of the ${ }^{8} \mathrm{~B}$ ground state in the case of one-channel models. Taking Kim model as a reference, we can see that the extracted $S_{17}$ factors for different g.s. models differ by less than $5 \%$.

When introducing deformation one could naïvely expect no change in the relationship between the extracted S-factor and the $\mathrm{ANC}$ of the ${ }^{8} \mathrm{~B}$ g.s. wave function. The ${ }^{8} \mathrm{~B}$ g.s. wavefunction can be written as $|\Psi\rangle=\alpha_{1}\left|\Psi_{p_{1 / 2}}\right\rangle\left|{ }^{7} \mathrm{Be}_{\text {g.s. }}\right\rangle+\alpha_{2}\left|\Psi_{p_{3 / 2}}\right\rangle\left|{ }^{7} \mathrm{Be}_{\text {g.s. }}\right\rangle$ and as both radial functions $\Psi_{p_{1 / 2}}$ and $\Psi_{p_{3 / 2}}$ have the same asymptotic behaviour, we have $b_{1 / 2}=b_{3 / 2}$. Given that the ${ }^{8} \mathrm{~B}$ wave function is normalized, $\alpha_{1}^{2}+\alpha_{2}^{2}=1$. Therefore, whatever the deformation, the single particle normalization constant is the same: $b^{2}=b_{1 / 2}^{2}=b_{3 / 2}^{2}$. When including excitation, since the contribution of the excited states decay rapidly to zero their contribution to the $S_{17}$ factor is expected to be negligible, as found in 11], and thus their contribution can be neglected when evaluating $b$ with eq.(7). As shown in table [ the ANC eq.(6) of $\mathrm{Xu}$ et al. for extracting the $S_{17}$ remains valid.

In contrast to the results shown in Table $\mathbb{I}$, we find that the DWBA cross section and therefore the S-factor are sensitive to the deformation and/or excitation for strong surface peaked optical potentials in the entrance channel. This can be seen in Table $\mathbb{1}$, where we compare the extracted S-factor using an optical potential for the entrance channel 25] that is strongly surface peaked, (here denoted by Surface II), with the results obtained using the Surface I optical potential. The $\chi^{2}$ is defined according to $\chi^{2}=\frac{1}{N_{\exp }} \sum_{i} \sqrt{\left(\frac{S_{\exp }(i)-S_{\operatorname{Theo}}(i)}{\Delta S_{\exp }(i)}\right)^{2}}$ 
with $N_{\text {exp }}$ the number of experimental points and $\Delta S_{\text {exp }}(i)$ the experimental error associated with each point. For no deformation or excitation, the extracted S-factor is essentially model independent for both optical potentials. In contrast, the extracted $S_{17}$ with the reo1 model (which has a larger component for the $\left[p_{3 / 2} \otimes \frac{3}{2}\right]$ channel) differs from the Kim model by only $1.4 \%$ for Surface I, but $16 \%$ for Surface II. The effects are similar for exc1 which has as well a larger component for the $\left[p_{3 / 2} \otimes \frac{3}{2}\right]$ channel. This is due to the fact that deformation modifies the low partial waves that are contributing significantly to the cross section. These modifications are probed by optical potentials which are strongly surface peaked for the incoming channel and have less interior absorption.

\section{Multistep processes}

The DWBA formalism assumes that the inelasticity arising from core excitation of the projectile and/or excitation of either projectile or target, has a small effect on the transfers. The low lying excited states of ${ }^{7}$ Be could in principle be excited by the deuteron prior to the transfer, giving rise to multiple step processes. In order to estimate the contribution of these processes to the differential cross section, we consider that the core $\left({ }^{7} \mathrm{Be}\right)$ is deformed, and consider quadrupole rotational nuclear couplings between its 3 bound states. In doing so, the transfer process will depend on couplings to and from the g.s. of ${ }^{7} \mathrm{Be}$ and the excited states, in addition to the transfer couplings themselves.

A diagram of the allowed 1, 2 and 3-step paths, excluding back transfer couplings, is shown in fig.(6). The observables were calculated including all couplings between the 3 states of ${ }^{7} \mathrm{Be}$ and transfer couplings between the 3 states of ${ }^{7} \mathrm{Be}$ and the g.s. of ${ }^{8} \mathrm{~B}$. We used Surface I optical potential for the entrance and the potential defined in [26] for the exit channel.

Fig.(77a) shows that the 2-step corrections to the elastic cross section calculated with model exc1 from [11]) are small, being evident only at large angles and that higher order multiple step corrections are small. Fig.(7) $\mathrm{b}$ ) also shows that the transfer cross section is not strongly sensitive to the multistep effects. They reduce the differential cross section peak by $\sim 8 \%$. As a direct consequence, the effect on the extracted $S_{17}$-factor (evaluated by renormalizing $S_{17}^{\text {cap }}$ by the experimental spectroscopic factor extracted from the transfer reaction) is around $\sim 8 \%$. This same result was confirmed using other core deformation/excitation models defined in [11] and with other optical potentials for the incoming and outgoing channel.

It is known that when introducing back couplings to the original DWBA specification, the optical potential fit to the scattering data may well be lost [35]. In fig.(8) we show the elastic scattering fig.(8a) and transfer cross section fig.(8) for 3 cases: a) all couplings between the core states are included but no back transfer couplings ; b) all couplings including back transfer couplings; c) no back transfer couplings and only up couplings for the core. As shown in the figure, the effect of introducing back transfer couplings is of same order of magnitude as the couplings from the excited states to the g.s. of ${ }^{7} \mathrm{Be}$.

The uncertainty arising from losing the elastic scattering fit, when introducing back transfer couplings, may be circumvented if we assume that these couplings are already effectively included in the optical potentials, and, to avoid double counting, should therefore be removed from our transfer calculations. This result calls once more for measuring the 
elastic scattering channels if one wants to avoid evaluating theoretically the second order coupling between the elastic and transfer channels.

\section{CONCLUSIONS}

We analyzed the transfer reaction ${ }^{7} \mathrm{Be}(\mathrm{d}, \mathrm{n})^{8} \mathrm{~B}$ for the recent experimental energies using DWBA. Checks on the ANC method for extracting the $S_{17}$ astrophysical factor from the transfer reaction were performed. We have shown that the lack of experimental data for the elastic scattering incoming and outgoing channels induces severe uncertainties on the extraction of the astrophysical factor. From the low energy transfer data of [14] we obtain a range for the S-factor $S_{17}(0)=23.5 \pm 3.7 \mathrm{eV}$ b based on using only potential parameters taken from elastic scattering at the correct energy.

We have shown that caution should be taken when extracting $S_{17}$ at center of mass energies approximately greater than $15 \mathrm{MeV}$ due to the fact that the transfer reaction cannot be considered as peripheral at these energies, given the angular range usually measured.

We have also shown that the $S_{17}$-factor depends upon the 2-body description of the ${ }^{8} \mathrm{~B}$ ground state wavefunction if deformation and/or excitation are included, for optical potentials that are strongly surface peaked, otherwise the extracted $S_{17}$-factor is essentially independent of the 2-body description of ${ }^{8} \mathrm{~B}$.

Finally we find multistep effects to the DWBA to be of the order of $8 \%$.

We conclude that the ANC method to extract the S-factor from transfer using the DWBA is accurate to $8 \%$ if the optical potentials for the incoming and outgoing channels are well defined, have no strong surface peaked potentials, and the reaction is clearly peripheral. More measurements at the appropriate low energy regime will help to further validate the method.

\section{ACKNOWLEDGMENTS}

This work was supported by Fundação de Ciência e Tecnologia (Portugal) through grant Praxis PCEX/C/FIS/4/96, and by EPSRC (U.K.) through grant GR/J95867. One of the authors, F. Nunes, was supported by FCT grant BIC 1483. We would like also to thank Didier Beaumel for providing us with references to optical potentials. 


\section{REFERENCES}

[1] E.G. Adelberger et al., Rev. Mod. Phys. 70 (1998) 1265

[2] Nuclear Physics limits to the ${ }^{8} B$ Solar Neutrino flux and the Kamiokande Experiment R. Crespo, A.M. Mourão, F.M. Nunes, to appear in Proceedings of the Workshop on New Worlds in Astroparticle Physics, Faro, Portugal, 8-10 September, 1996.

[3] R.W. Kavanagh, Nucl. Phys. 15 (1960) 411.

[4] F.J. Vaughn, R.A. Chalmers, D. Kohler and L.F. Chase Jr, Phys Rev. C2 (1970) 1657.

[5] B.W. Filippone, A.J. Elwyn, C.N. Davids and D.D. Koetke, Phys. Rev. Lett. 50 (1983) 412; Phys. Rev. C28 (1983) 2222.

[6] P.D. Parker, Astrophys. J. (Lett) L85 (1968) 153.

[7] R.W. Kavanagh, T.A. Tombrello, J.M. Mosher and D.R. Goosman, Bull. Am. phys. Soc. 14 (1969) 1209.

[8] C. Wiezorek, H. Krawinkel, R. Santo and K. Wallek, Z. Phys A282 (1977) 121.

[9] F. Hammache et al., Phys. Rev. Lett. 80 (1998) 928.

[10] F.M. Nunes, I.J. Thompson and R.C. Johnson, Nucl. Phys. A596 (1996) 171.

[11] F.M. Nunes R. Crespo and I.J. Thompson, Nucl. Phys. A615 (1997) 69; Nucl. Phys. A627 (1997) 747.

[12] A. Csótó and K.-H. Langanke, Nucl. Phys. A636 (1998) 240.

[13] H.M. Xu, C.A. Gagliardi, R.E. Tribble, A.M. Mukhamedzhanov and N.K. Timofeyuk, Phys. Rev. Lett. 73 (1994) 2027

[14] W. Liu et al, Phys. Rev. Lett. 77 (1996) 611.

[15] C. Powell et al., Study of the $d\left({ }^{7} B e,{ }^{8} B\right) n$ reaction AIP Proceedings of the 2nd International Conference on Exotic Nuclei and Atomic Masses, ENAM98.

[16] M. Ishihara et al, Determination of the Astrophysical S-Factor for the ${ }^{7} \mathrm{Be}(\mathrm{p}, \gamma)^{8} \mathrm{~B}$ capture from the ${ }^{7} \mathrm{Be}(\mathrm{d}, \mathrm{n})^{8} \mathrm{~B}$ reaction, Proposal for Nuclear Physics Experiment at RIKEN Ring Cyclotron, July 1996.

[17] I.J. Thompson, Comp. Phys. Rep., 7 (1988) 167.

[18] B.K. Jennings, S. Karataglidis and T.D. Shoppa, Phys. Rev. C58 (1998) 579.

[19] F.C. Barker, Aust. J. Phys. 33 (1980) 177.

[20] F. Ajzenberg-Selove, Nucl. Phys. A 490 (1988) 1.

[21] K.H. Kim, M.H. Park and B.T. Kim, Phys. Rev. C35 (1987) 363.

[22] T.A. Tombrello, Nucl. Phys. 71 (1965) 459

[23] R.G. Robertson, Phys. Rev. C 7 (1973) 543

[24] H. Esbensen and G.F. Bertsch, Nucl. Phys. A 600 (1996) 66.

[25] G.R. Satchler, Private communication to P.E.Hodgson (1965), see C.M. Perey and F.G. Perey, Atomic Data and Nuclear Data tables, 171 (1976).

[26] J.H. Dave and C.R. Gould, Phys. Rev. C28 (1983) 2212.

[27] M. El-Nadi et al., Ann. Phys. (Leipzig) 25 (1970) 1.

[28] N. Olsson, E. Ramstromm and B. Trostell, Nucl. Phys. A 509, 161 (1990).

[29] J.S. Al-Khalili and J.A. Tostevin, Phys. Rev. Letts. 76 (1996) 3903.

[30] C.A. Gagliardi et al., Phys. Rev. Letts. 80 (1998) 421.

[31] J.C. Fernandes, R. Crespo, F.M. Nunes and I.J. Thompson Determining the astrophysical $S_{17}$ with transfer reactions, AIP Proceedings of the $2^{\text {nd }}$ International Conference on Exotic Nuclei and Atomic Masses, ENAM98

[32] H. Ludecke, Tan Wan-Tjin, H. Werner and J. Zimmerer, Nucl. Phys. A109 (1968) 676. 
[33] H.G. Bingham, A.R. Zander, K.W. Kemper and N.R. Fletcher, Nucl. Phys. A173(1971)265.

[34] S. Matsuki et al., J. Phys. Soc. Japan 26 (1969) 1344.

[35] G.R. Satchler, Direct Nuclear Reactions, Clarendon Press, Oxford 1983 


\section{TABLES}

TABLE I. Ratio of the asymptotic normalization constant of the w.f to the direct capture S-factor at $20 \mathrm{KeV}$.

\begin{tabular}{cccc}
\hline \hline Model & $\begin{array}{c}b^{2} \\
\left(\mathrm{fm}^{-1}\right)\end{array}$ & $\begin{array}{c}S_{17}^{\text {cap }} \\
(\mathrm{eV} \mathrm{b})\end{array}$ & $\begin{array}{c}b^{2} / S_{17}^{\text {cap }} \\
\left(\mathrm{eV}^{-1} \mathrm{~b}^{-1} \mathrm{fm}^{-1}\right)\end{array}$ \\
\hline Kim & 0.6354 & 23.8 & 0.0267 \\
Tombrello & 0.6279 & 23.5 & 0.0267 \\
Robertson & 0.6426 & 24.0 & 0.0268 \\
Barker & 0.5946 & 22.4 & 0.0265 \\
Esbensen & 0.4972 & 18.8 & 0.0264 \\
\hline reor1 & 0.6710 & 25.2 & 0.0267 \\
reor2 & 0.6967 & 26.0 & 0.0268 \\
exc1 & 0.5810 & 22.0 & 0.0264 \\
exc2 & 0.6265 & 24.1 & 0.0260 \\
\hline \hline
\end{tabular}

TABLE II. Comparing the $S_{17}^{I}$ factors extracted from transfer data at $\mathrm{E}_{c m}=5.8 \mathrm{MeV}$ with those obtained using direct capture calculations $S_{17}^{I I}$, for different ${ }^{8} \mathrm{~B}$ g.s models.

\begin{tabular}{|c|c|c|c|c|c|c|c|c|}
\hline \multirow[b]{2}{*}{ Model } & \multicolumn{4}{|c|}{ Surface I } & \multicolumn{4}{|c|}{ Surface II } \\
\hline & $\mathcal{S}_{\text {exp }}$ & $\chi^{2}$ & $\begin{array}{c}S_{17}^{I} \\
(\mathrm{eV} \text { b) }\end{array}$ & $\begin{array}{c}S_{17}^{I I} \\
(\mathrm{eV} \mathrm{b})\end{array}$ & $\mathcal{S}_{\text {exp }}$ & $\chi^{2}$ & $\begin{array}{c}S_{17}^{I} \\
(\mathrm{eV} \mathrm{b})\end{array}$ & $\begin{array}{c}S_{17}^{I I} \\
(\mathrm{eV} \mathrm{b})\end{array}$ \\
\hline $\mathrm{Kim}$ & 1.076 & 0.60 & 26.30 & 25.61 & 0.822 & 0.69 & 20.10 & 19.57 \\
\hline Tombrello & 1.048 & 0.69 & 25.31 & 24.63 & 0.832 & 0.69 & 20.10 & 19.55 \\
\hline Robertson & 1.017 & 0.72 & 25.14 & 24.41 & 0.813 & 0.70 & 20.10 & 19.52 \\
\hline Barker & 1.138 & 0.58 & 26.03 & 25.50 & 0.881 & 0.66 & 20.15 & 19.74 \\
\hline Esbensen & 1.402 & 0.46 & 26.82 & 26.36 & 1.041 & 0.63 & 19.91 & 19.57 \\
\hline reor1 & 1.005 & 0.54 & 25.94 & 25.32 & 0.652 & 0.73 & 16.83 & 16.43 \\
\hline reor2 & 0.968 & 0.64 & 25.93 & 25.16 & 0.731 & 0.72 & 19.59 & 19.01 \\
\hline exc1 & 1.170 & 0.54 & 26.15 & 25.75 & 0.749 & 0.67 & 16.73 & 16.47 \\
\hline exc2 & 1.099 & 0.60 & 26.47 & 26.47 & 0.862 & 0.65 & 20.77 & 20.77 \\
\hline
\end{tabular}




\section{FIGURES}
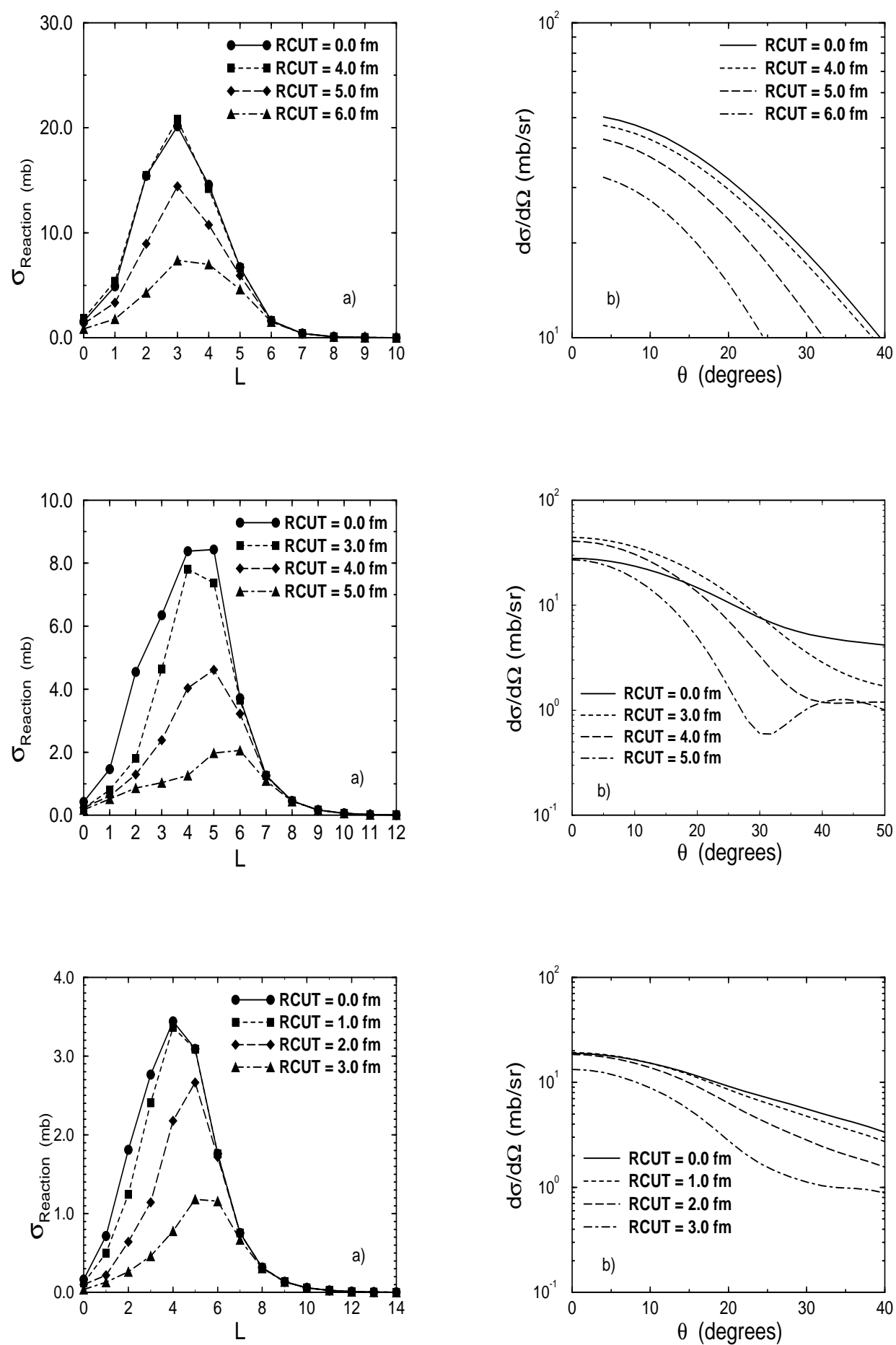

FIG. 1. Calculated reaction cross section in the partial wave $\mathrm{L}$, for the $\left.{ }^{7} \mathrm{Be}(\mathrm{d}, \mathrm{n}){ }^{8} \mathrm{~B}\right)$ at $5.8,15.6$ and $38.9 \mathrm{MeV}$ c.m. energies (a) and corresponding differential cross section (b). 

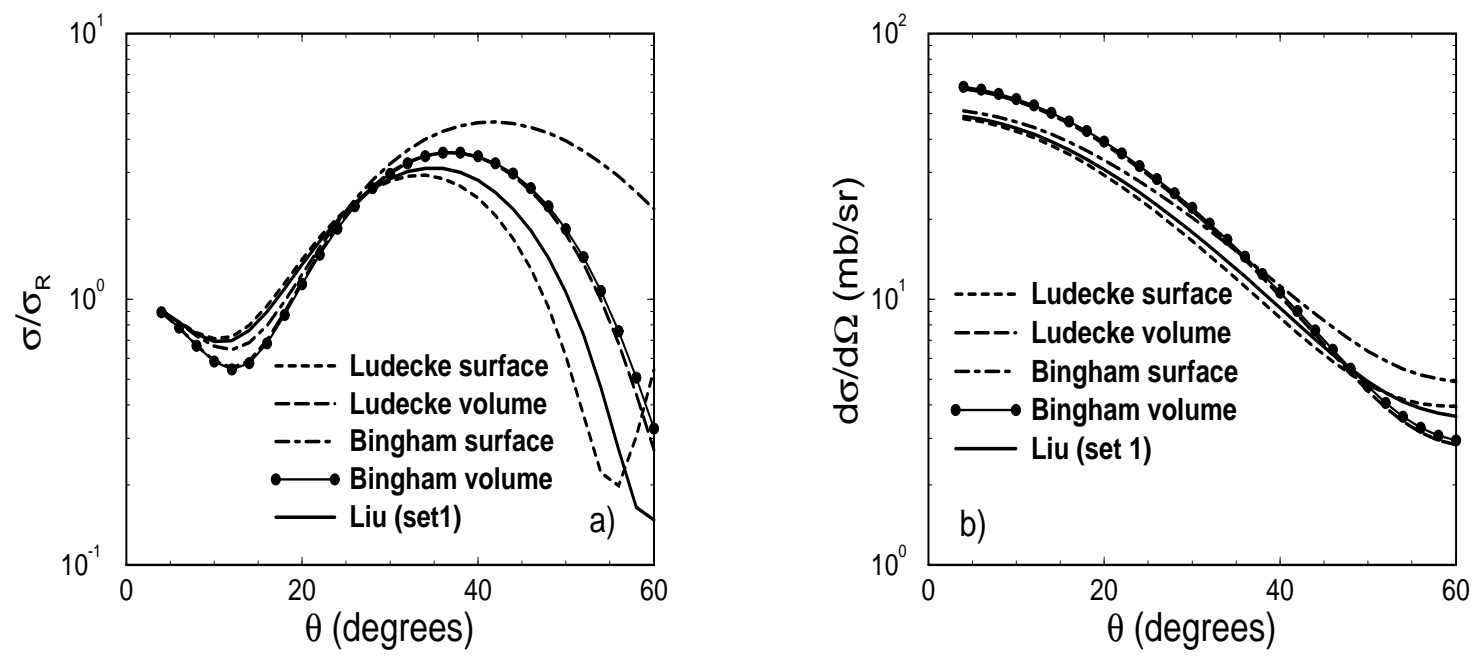

FIG. 2. The d- ${ }^{7}$ Be elastic scattering at $5.8 \mathrm{MeV}$ for a set of optical potentials taken from fits to $\simeq 9.3 \mathrm{MeV}$ data (a) and the corresponding transfer differential cross sections (b).
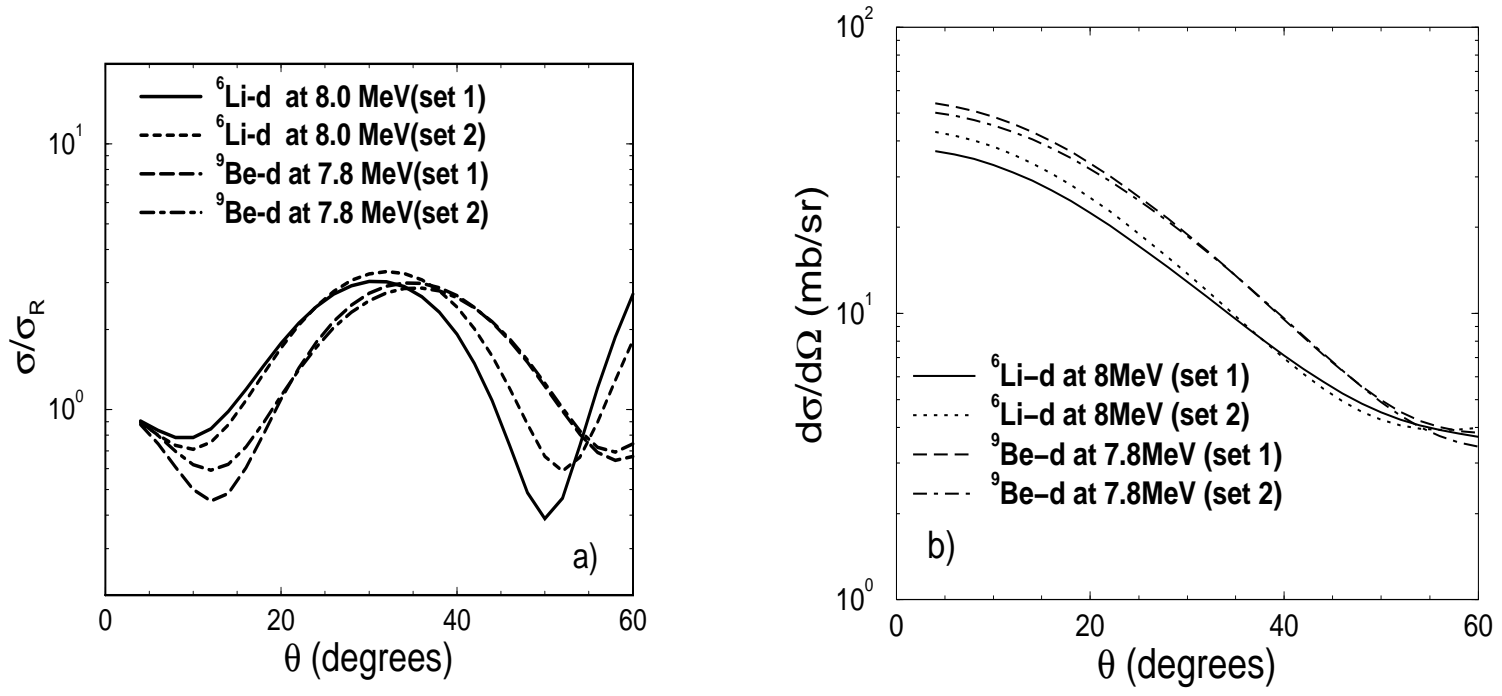

FIG. 3. The $\mathrm{d}^{7} \mathrm{Be}$ elastic scattering at $5.8 \mathrm{MeV}$ for a set of optical potentials taken at the correct energy but on different targets (a) and the corresponding transfer differential cross sections (b). 

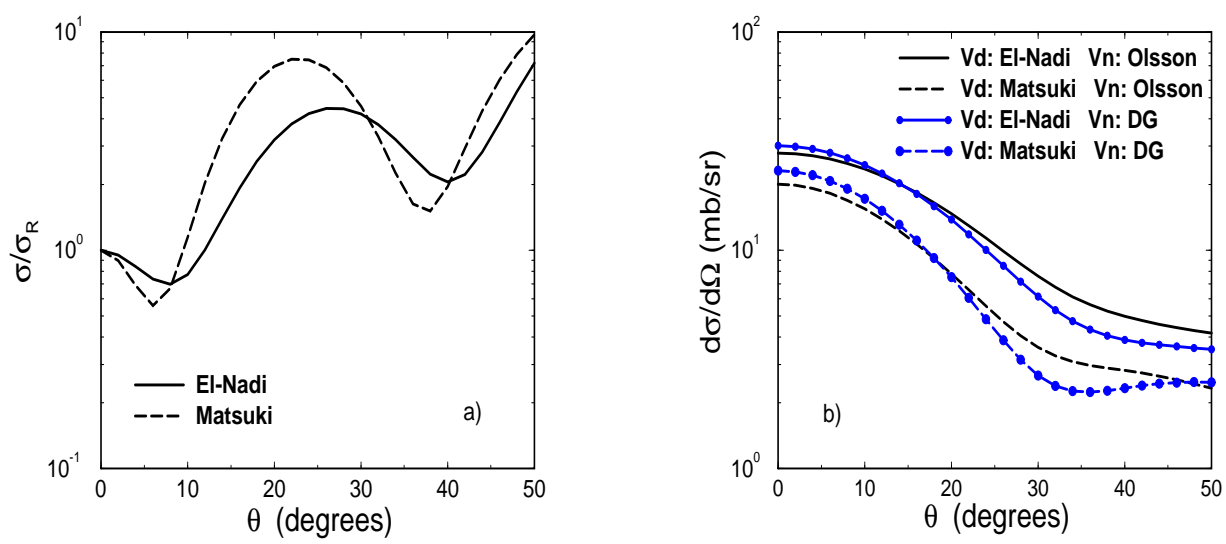

FIG. 4. The $\mathrm{d}-{ }^{7}$ Be elastic cross section (a) calculated at $\mathrm{E}_{\mathrm{cm}}=15.6 \mathrm{MeV}$ for two sets of optical potentials (see text), and the corresponding transfer differential cross sections (b).

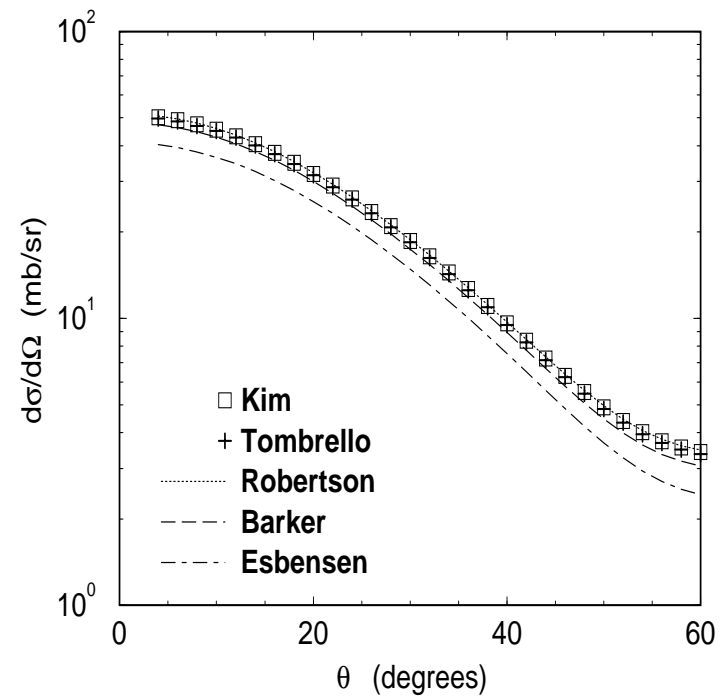

FIG. 5. Calculated differential cross section for the ${ }^{7} \mathrm{Be}(\mathrm{d}, \mathrm{n})^{8} \mathrm{~B}$ reaction using different models for the ${ }^{8} \mathrm{~B}$ g.s. at $\mathrm{E}_{\mathrm{cm}}=5.8 \mathrm{MeV}$. 


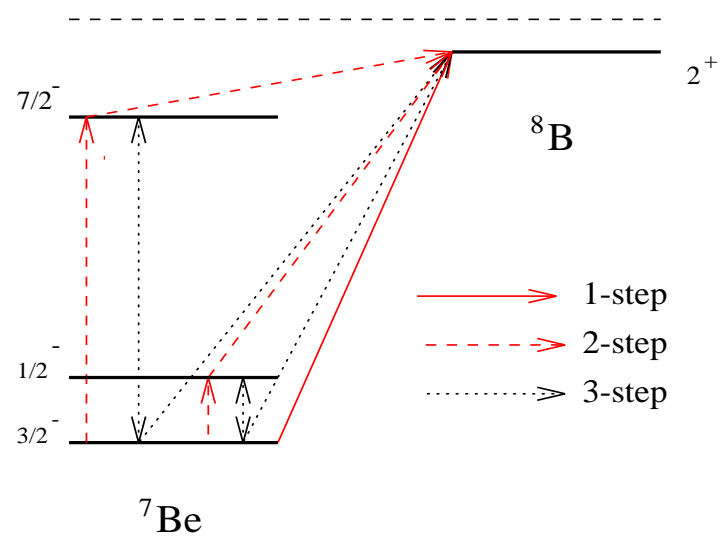

FIG. 6. The coupling diagram for multistep processes excluding transfer back couplings.
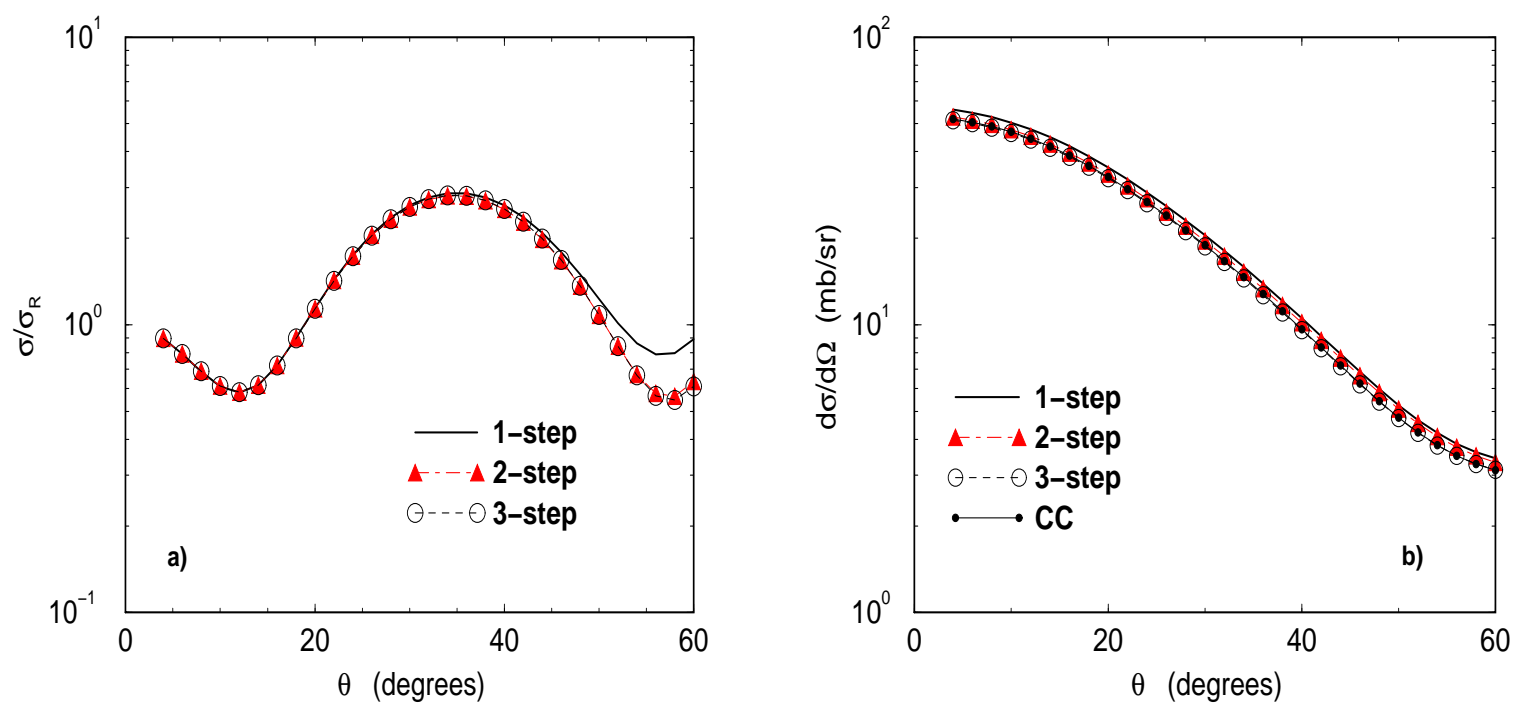

FIG. 7. a) The elastic scattering (a) and the transfer cross section (b) for 1-step, 2-step, 3-step and inelastic coupled channel calculations at $\mathrm{E}_{\mathrm{cm}}=5.8 \mathrm{MeV}$. 

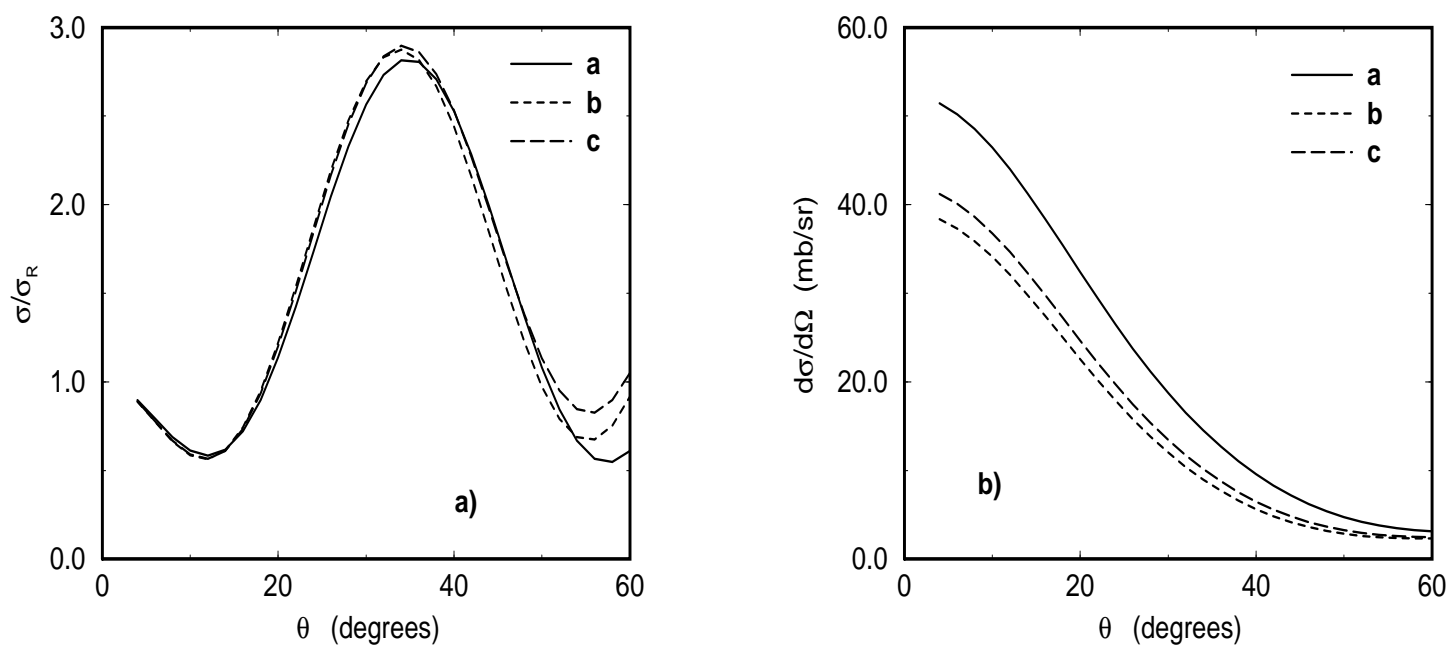

FIG. 8. Comparing the $d-{ }^{7}$ Be elastic scattering (a) and transfer reaction (b) when different types of couplings (see text) are included at $\mathrm{E}_{\mathrm{cm}}=5.8 \mathrm{MeV}$. 